\title{
FACTORES PERSONALES Y ESCOLARES SUBYACENTES A LA CONDUCTA AUTODETERMINADA DE NIÑOS Y ADOLESCENTES CON DISCAPACIDAD INTELECTUAL: REVISIÓN DE LA LITERATURA
}

\section{Personal and School Factors Underlying the Self-Determined Behaviour of Children and Adolescents: Review of the Literature}

Leticia BlázQuez Arribas

Universidad de Salamanca. Facultad de Educación

leticia.blaz@usal.es

María Isabel Calvo Álvarez

Universidad de Salamanca. Facultad de Educación. Instituto Universitario de Integración en la Comunidad

María Begoña Orgaz BAz

Universidad de Salamanca. Facultad de Psicología. Instituto Universitario de Integración en la Comunidad

Recepción: 10 de junio de 2020

Aceptación: 14 de mayo de 2021

RESUMEN: La autodeterminación es una característica disposicional que se pone de manifiesto cuando la persona tiene cierto control sobre su propia vida. Se adquiere a lo largo de la vida en interacción con el entorno, de ahí la importancia de evaluar los factores contextuales que pueden influir en ella. El objetivo general de este trabajo es conocer las publicaciones de los últimos años que han investigado los factores contextuales que pueden influir en la autodeterminación de jóvenes estudiantes con discapacidad intelectual. Se analizan diecinueve artículos y dos tesis doctorales publicados durante el periodo 
FACTORES PERSONALES Y ESCOLARES SUBYACENTES A LA CONDUCTA AUTODETERMINADA

DE NIÑOS Y ADOLESCENTES CON DISCAPACIDAD INTELECTUAL: REVISIÓN DE LA LITERATURA L. BLÁZQUEZ ARRIBAS, M. ${ }^{a}$ I. CALVO ÁlVAREZ Y M. ${ }^{a}$ B. ORGAZ BAZ

2015-2020. Todos ellos utilizan una metodología cuantitativa, excepto dos trabajos que utilizan una metodología cualitativa. Los estudios señalan resultados dispares respecto a las variables personales, como el género, la edad o el CI de los participantes. Respecto a las variables escolares, se consideran las diferencias entre la educación ordinaria y la educación especial; sin embargo, los apoyos educativos adquieren mayor significación en la promoción de la autodeterminación. En definitiva, las estrategias utilizadas en el aula y los apoyos individualizados influyen en la autodeterminación de este colectivo.

Palabras Clave: autodeterminación; calidad de vida; discapacidad intelectual; adolescencia; apoyos educativos.

AвSTRACT: Self-determination is a dispositional characteristic that is evident when the person has certain control over their own lives. It is acquired throughout life in interaction with the environment, hence the importance of evaluating the contextual factors that can influence it. This study aims to review publications from the last years that have investigated the contextual factors that can influence the self-determination of young students with intellectual disabilities. Nineteen articles and two doctoral theses published during the 2015-2020 were analyzed. All of them use a quantitative methodology, except for two studies that use a qualitative methodology. The studies indicate mixed results regarding personal variables, such as gender, age or IQ. Regarding school factors, the differences between regular and special education are considered. However, educational supports acquire more significance to promote self-determination. In the end, the strategies used in the classroom and individualized student-supports show an influence in the self-determination of this group.

KEYWORDS: self-determination; quality of life; intellectual disability; adolescence; educational support.

\section{Introducción}

egún la Convención sobre los Derechos de las Personas con Discapacidad, es necesario estudiar las dificultades y los apoyos que se encuentran las personas con discapacidad intelectual (en adelante DI) para vivir según sus deseos y para que se tengan en cuenta sus experiencias y opiniones, es decir, para vivir con autodeterminación.

Desde la década de 1990 han existido diferentes teorías que explican la autodeterminación (Mumbardó-Adam et al., 2018). Hoy en día, la Teoría de la Agencia Causal concibe la autodeterminación como "una característica disposicional que se pone de manifiesto cuando la persona actúa como agente causal en la propia vida. Las personas autodeterminadas actúan en servicio de sus metas libremente elegidas" (Shogren et al., 2015, p. 258). En otras palabras, son responsables de los acontecimientos que les suceden; son autónomas y empoderadas; se conocen bien a sí mismas, y, además, inician, dirigen y autorregulan acciones para lograr el objetivo deseado, en función de sus preferencias y necesidades (DiMaggio et al., 2020; Shogren et al., 2017). 
FACTORES PERSONALES Y ESCOLARES SUBYACENTES A LA CONDUCTA AUTODETERMINADA

DE NIÑOS Y ADOLESCENTES CON DISCAPACIDAD INTELECTUAL: REVISIÓN DE LA LITERATURA L. BLÁZQUEZ ARRIBAS, M. ${ }^{a}$ I. CALVO ÁlVAREZ Y M. ${ }^{a}$ B. ORGAZ BAZ

La autodeterminación es considerada una de las dimensiones que forman el concepto de Calidad de Vida (en adelante CV) de Schalock y Verdugo (2002/2003), el cual permite identificar necesidades, planificar apoyos y dar respuesta a las dificultades del día a día (Schalock, 2010).

En los últimos años, las prácticas para promover la conducta autodeterminada están siendo incorporadas progresivamente en el contexto educativo para asegurar una educación inclusiva, equitativa y de calidad que promueva las oportunidades de aprendizaje de todas las personas durante toda la vida (Unesco, 2016), y, así, enfrentar las demandas sociales propias de la adolescencia y de la transición a la vida adulta (Álvarez-Aguado et al., 2020; Vicente et al., 2018). La importancia de la conducta autodeterminada en la adolescencia radica en el hecho de que los estudiantes más autodeterminados presentan mayor probabilidad de obtener resultados escolares positivos, óptima transición a la vida adulta, disfrute del ocio y tiempo libre, buenas relaciones interpersonales, participación en la comunidad y una mayor CV (CudréMauroux et al., 2020; DiMaggio et al., 2020; Wehmeyer, 2020).

No obstante, investigaciones actuales indican que jóvenes y adolescentes con DI no disfrutan de un alto grado de autodeterminación, ni de CV en general, comparándolos con sus iguales sin discapacidad (Garrels y Granlund, 2018; Muñoz-Cantero et al., 2018). La literatura explica que, en general, los adolescentes con DI carecen de habilidades, conocimientos y creencias que son importantes para su autodeterminación, por ejemplo, tienen dificultades para regular sus emociones y tomar decisiones importantes, $\mathrm{y}$, además, demuestran escasas habilidades de autoconocimiento y de autodefensa (Álvarez-Aguado et al., 2020).

Considerar que la mayoría de adolescentes con DI no actúan con autodeterminación conlleva la necesidad de analizar las variables que pudiesen tener relación con esta conducta (Wehmeyer, 2006). La revisión de la literatura llevada a cabo por BañaCastro et al. (2015) reflejó que la autodeterminación está formada por la interacción de factores contextuales, los cuales se dividen en factores personales (por ejemplo, el género, aunque la información es limitada y contradictoria; la edad, los autores concluyen que a mayor autodeterminación, mejores resultados en la vida adulta; y el cociente intelectual, considerando que la inteligencia no es en sí misma un factor determinante de la conducta autodeterminada) y factores ambientales (relacionados con la cultura, aunque no se encuentran resultados concluyentes, con la familia, explicando una compleja relación entre estatus socioeconómico, educación parental y autodeterminación, y una influencia de las creencias familiares en la adquisición de conductas autodeterminadas; y con la escuela, el estudio concluye que los jóvenes que asisten a centros específicos de educación especial presentan mayor grado de autodeterminación y, además, los datos determinan que el profesorado que trabaja con alumnado con discapacidad leve es más propenso a mostrar actitudes positivas frente a la importancia de la autodeterminación que aquellos que trabajan con alumnado con discapacidades más graves).

Partiendo de las conclusiones planteadas por Baña-Castro et al. (2015) y de la escasez de investigaciones que analizan dichos factores en personas con DI (Navas et al., 2017; Vicente et al., 2018), el objetivo general de este estudio es conocer las 
FACTORES PERSONALES Y ESCOLARES SUBYACENTES A LA CONDUCTA AUTODETERMINADA

DE NIÑOS Y ADOLESCENTES CON DISCAPACIDAD INTELECTUAL: REVISIÓN DE LA LITERATURA L. BLÁZQUEZ ARRIBAS, M. ${ }^{a}$ I. CALVO ÁlVAREZ Y M. ${ }^{a}$ B. ORGAZ BAZ

publicaciones de los últimos cinco años (2015-2020) que han investigado los factores contextuales que pueden influir en la autodeterminación de adolescentes con DI. Este trabajo permite conocer qué variables influyen en la autodeterminación para asegurar que todos los niños y adolescentes adquieran dicha conducta antes de que alcancen la vida adulta. Para ello se comprueba si existen diferencias en función de variables personales (género, edad y CI) y variables escolares (tipo de escolarización y apoyos educativos recibidos).

\section{Método}

Se realizó una búsqueda en las bases de datos más utilizadas en el campo de la investigación de las ciencias sociales y la psicología: Academic Search Complete, ERIC, PSICODOC, Psychology and Behavioral Sciences Collection, PsycINFO y SCOPUS. También se efectuó una búsqueda complementaria a través de Google Scholar y en los repositorios de diferentes universidades españolas con el fin de identificar trabajos académicos de los últimos años sobre la autodeterminación y la discapacidad.

\subsection{Procedimiento}

Se establecieron criterios de inclusión para la selección de los artículos de la revisión: 1) artículos empíricos; 2) artículos publicados entre 2015 y 2020; 3) participantes jóvenes y adolescentes con DI entre 11-21 años; 4) artículos que analizan los factores personales y ambientales que influyen en la autodeterminación; 5) artículos publicados en inglés o en español.

Los términos utilizados para la búsqueda con diferentes combinaciones, en inglés y en español, fueron elegidos de acuerdo al tema objeto del trabajo: "autodeterminación" ("self-determination"), "discapacidad intelectual” ("intelectual disability"), "factores contextuales" ("contextual factors"), "escuela" ("school”), "influencias ambientales" ("environment influences"), "características individuales" ("individual characteristics") y "calidad de vida" ("quality of life").

\subsection{Análisis de la información}

La búsqueda inicial obtuvo un total de 789 artículos, introduciendo "autodeterminación" ("self-determination") y "discapacidad intelectual" ("intelectual disability") como palabras clave y empleando el filtro temporal correspondiente (2015-2020).

Posteriormente, se tuvo en cuenta la población al que iba dirigida, eligiendo aquellos orientados a una población de niños y adolescentes y se combinaron el resto de palabras clave mencionadas anteriormente. Seguidamente, se descartaron los duplicados y se seleccionaron un total de 38 artículos, los cuales fueron de nuevo revisados atendiendo al título y a los resúmenes, con el fin de desechar aquellos que no tratasen 
de manera directa y empírica la influencia de factores contextuales sobre la conducta autodeterminada de niños y adolescentes con DI, objeto de la revisión. El siguiente paso fue realizar una lectura completa del texto. En la Figura 1 se presenta el proceso de selección de los artículos.

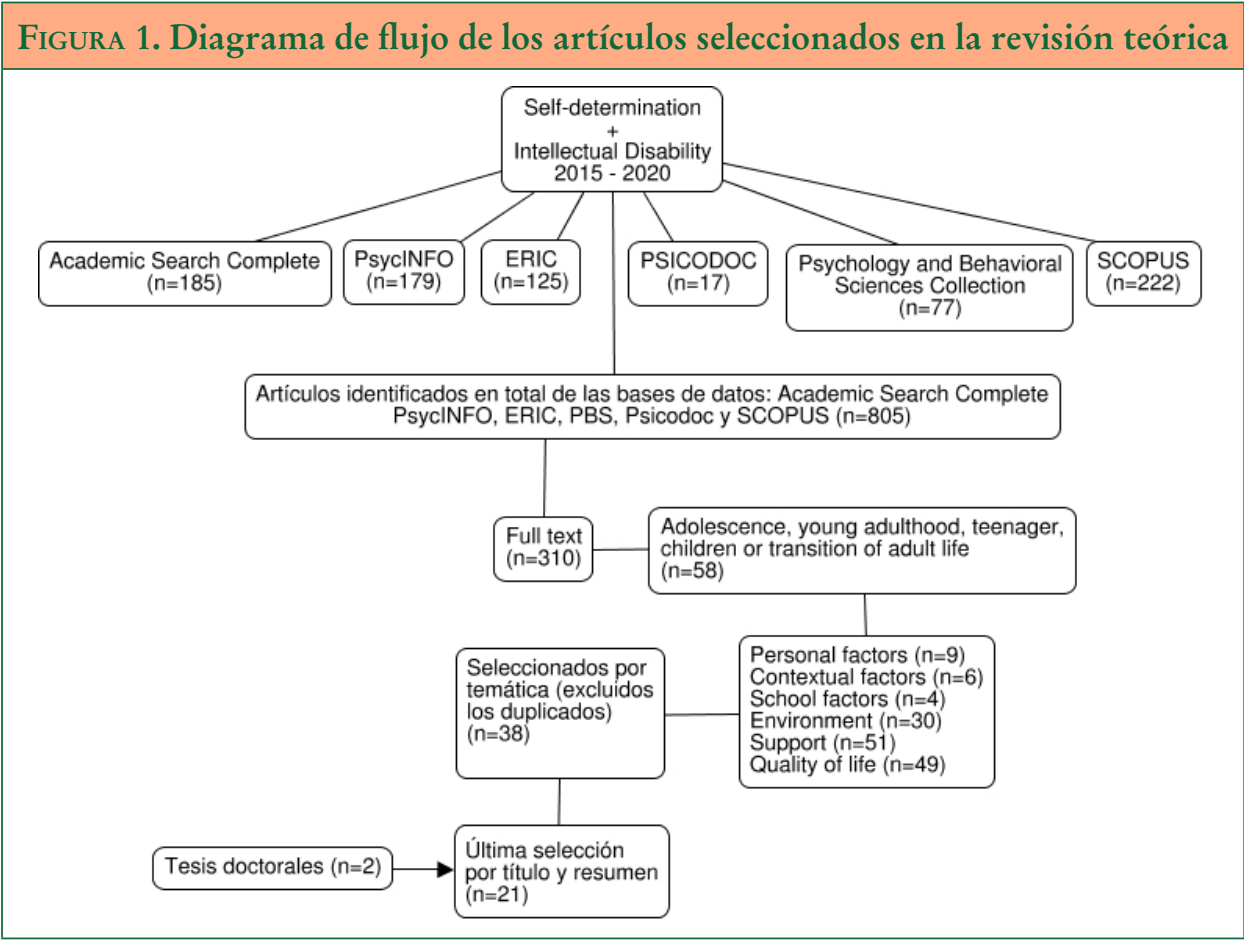

Fuente: Elaboración propia.

\section{Resultados}

Finalmente, siguiendo el proceso de inclusión y exclusión, se han seleccionado diecinueve artículos y dos tesis doctorales publicados durante el periodo 2015-2020. Ocho de ellos se desarrollan en el contexto español, siete en el contexto estadounidense y el resto corresponde a artículos publicados en Colombia, Chile, Suiza, Noruega y Rusia. Excepto cuatro artículos, todos ellos involucran al propio estudiante con DI en el proceso de evaluación para conocer su perspectiva sobre el constructo evaluado. No obstante, tres de estos trabajos evalúan no solo la percepción del estudiante, sino también la de su familia y la del equipo docente.

Los artículos seleccionados corresponden a un total de 16 revistas indexadas en SCOPUS, once de ellas también están indexadas en Web of Science. Todas ellas cuen- 
FACTORES PERSONALES Y ESCOLARES SUBYACENTES A LA CONDUCTA AUTODETERMINADA

DE NIÑOS Y ADOLESCENTES CON DISCAPACIDAD INTELECTUAL: REVISIÓN DE LA LITERATURA

L. BLÁZQUEZ ARRIBAS, M. ${ }^{a}$ I. CALVO ÁLVAREZ Y M. ${ }^{a}$ B. ORGAZ BAZ

tan con un proceso de revisión por pares que aseguran la calidad de las publicaciones. Respecto a las tesis doctorales, han sido recuperadas de los repositorios de la Universidad de A Coruña y de la Universidad de Salamanca, respectivamente. En la Tabla 1 se detallan los artículos seleccionados con sus principales características. A continuación, se presentan los resultados divididos en dos partes: variables personales $\mathrm{y}$ variables ambientales. 
FACTORES PERSONALES Y ESCOLARES SUBYACENTES A LA CONDUCTA AUTODETERMINADA DE NIÑOS Y ADOLESCENTES CON DISCAPACIDAD INTELECTUAL: REVISIÓN DE LA LITERATURA L. BLÁZQUEZ ARRIBAS, M. ${ }^{a}$ I. CALVO ÁlVAREZ Y M. ${ }^{a}$ B. ORGAZ BAZ

\begin{tabular}{|c|c|c|c|}
\hline 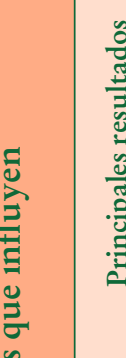 & 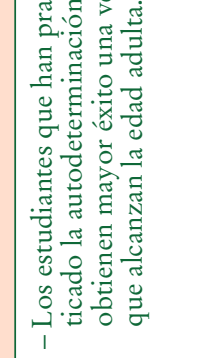 & 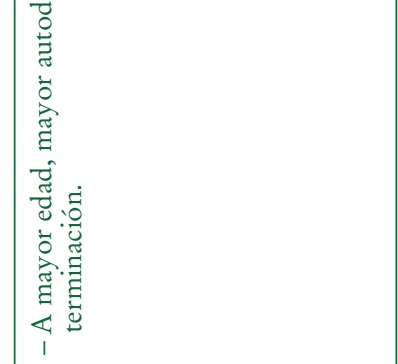 & 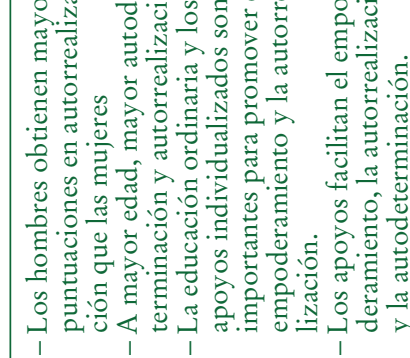 \\
\hline & 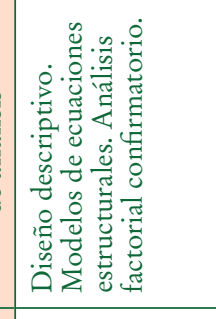 & 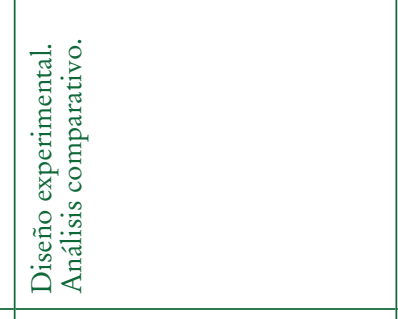 & 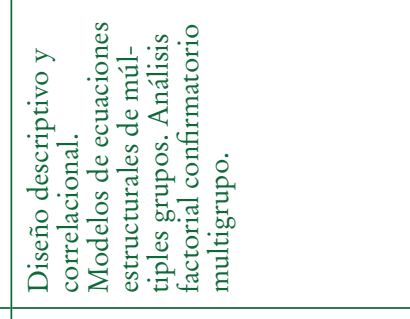 \\
\hline & 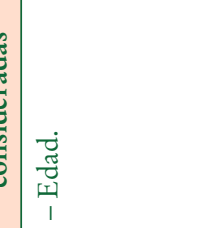 & 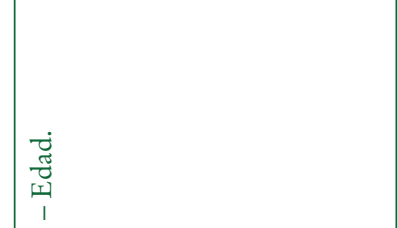 & 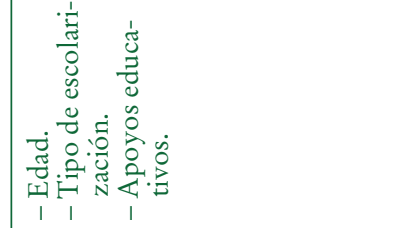 \\
\hline & 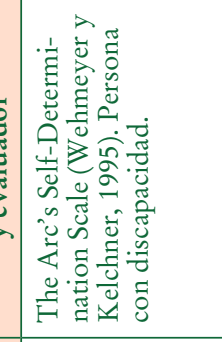 & 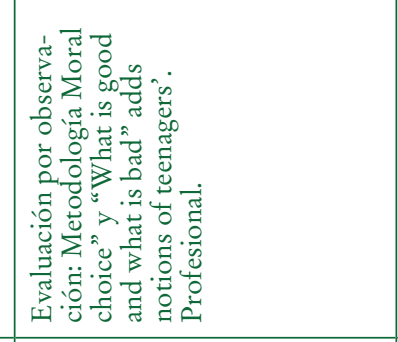 & 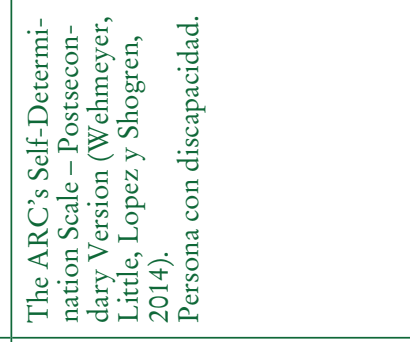 \\
\hline & 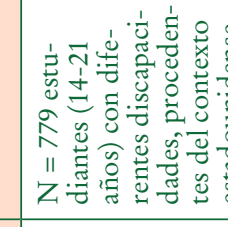 & 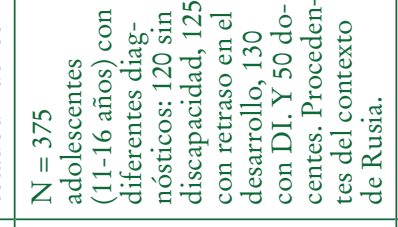 & 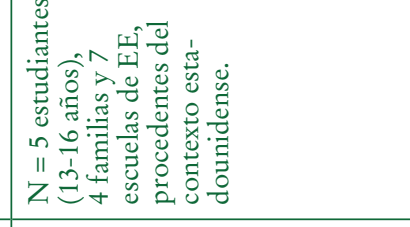 \\
\hline ( & 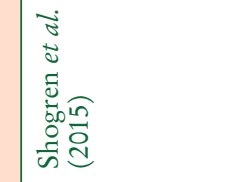 & 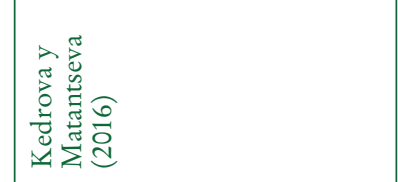 & 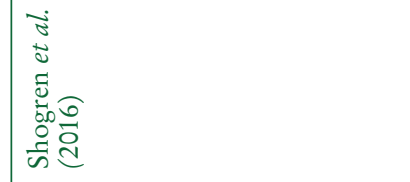 \\
\hline
\end{tabular}

Ediciones Universidad de Salamanca / CC BY-NC-ND

Siglo Cero, vol. 52 (4), 2021, octubre-diciembre, pp. 109-130 
FACTORES PERSONALES Y ESCOLARES SUBYACENTES A LA CONDUCTA AUTODETERMINADA DE NIÑOS Y ADOLESCENTES CON DISCAPACIDAD INTELECTUAL: REVISIÓN DE LA LITERATURA L. BLÁZQUEZ ARRIBAS, M. ${ }^{a}$ I. CALVO ÁlVAREZ Y M. ${ }^{a}$ B. ORGAZ BAZ

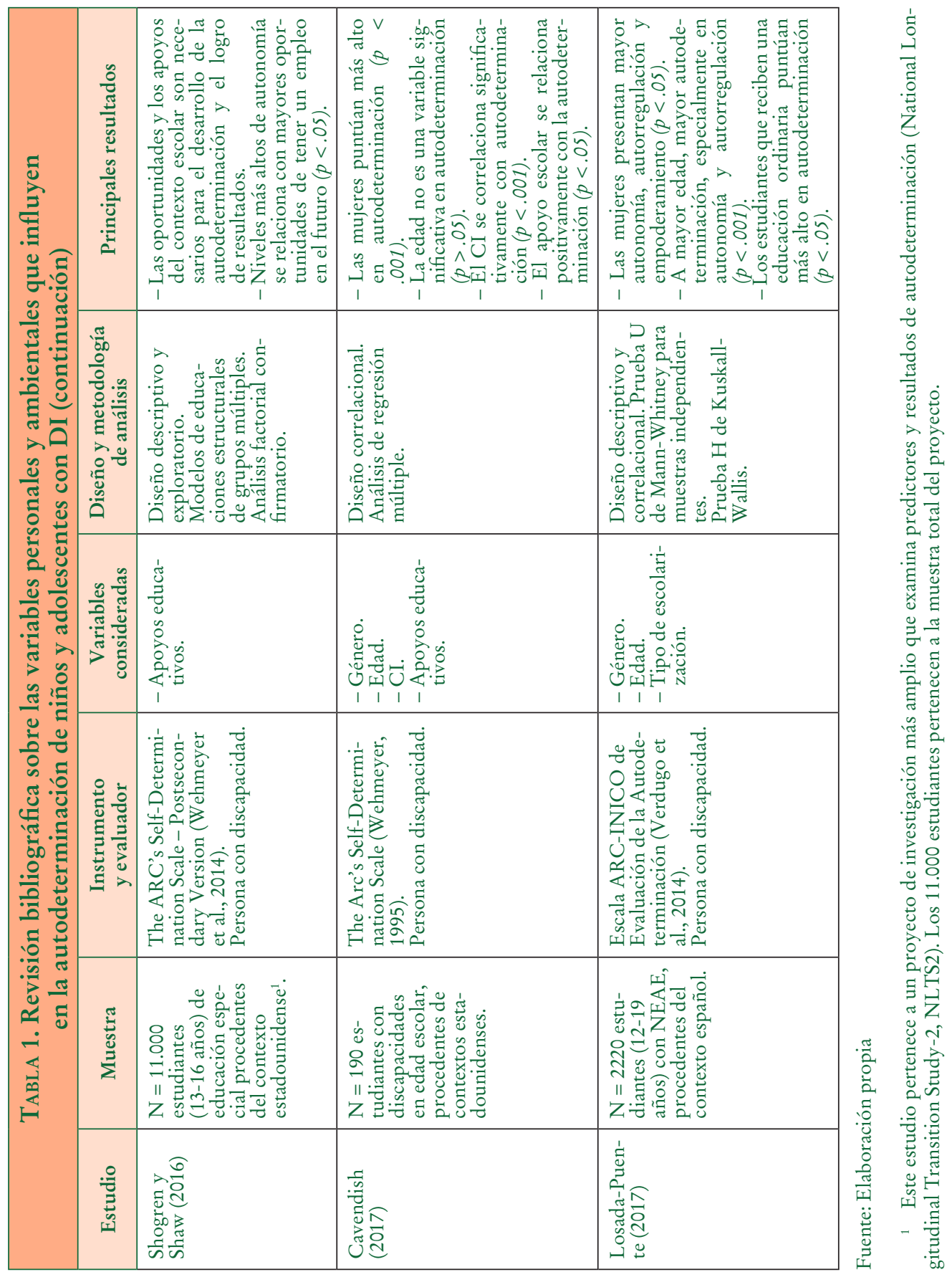

Ediciones Universidad de Salamanca / CC BY-NC-ND

Siglo Cero, vol. 52 (4), 2021, octubre-diciembre, pp. 109-130 
FACTORES PERSONALES Y ESCOLARES SUBYACENTES A LA CONDUCTA AUTODETERMINADA

DE NIÑOS Y ADOLESCENTES CON DISCAPACIDAD INTELECTUAL: REVISIÓN DE LA LITERATURA L. BLÁZQUEZ ARRIBAS, M. ${ }^{a}$ I. CALVO ÁlVAREZ Y M. ${ }^{a}$ B. ORGAZ BAZ

\begin{tabular}{|c|c|c|c|c|}
\hline \multirow{6}{*}{ 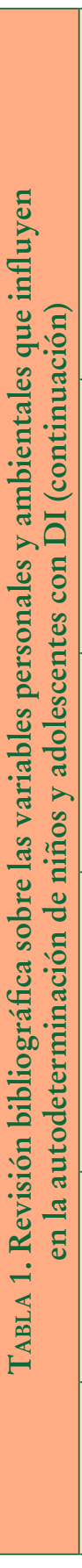 } & 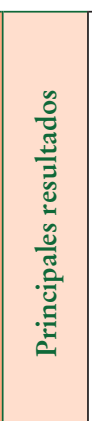 & 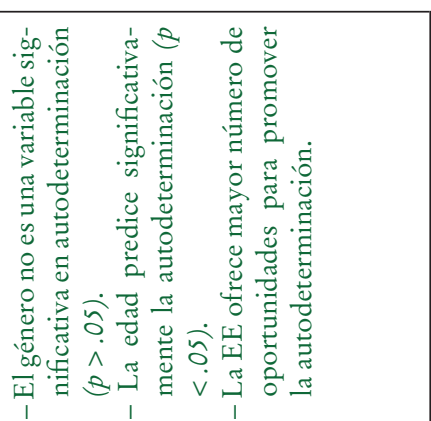 & 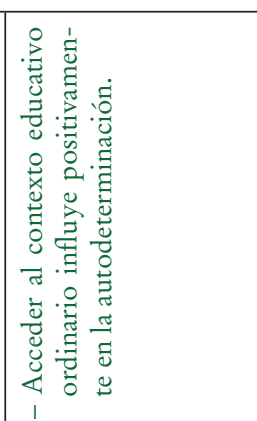 & 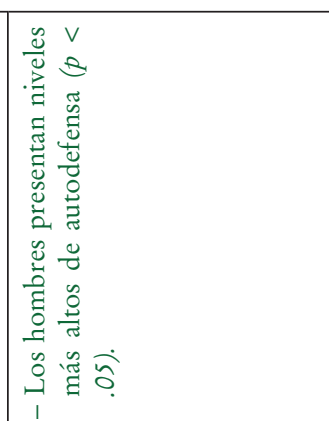 \\
\hline & 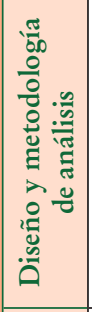 & 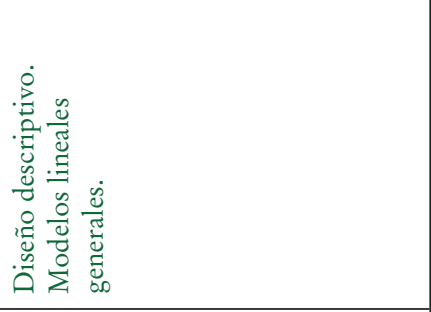 & 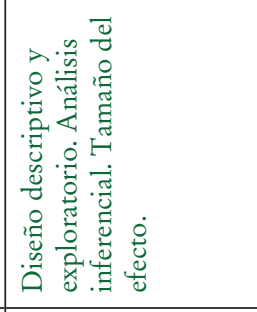 & 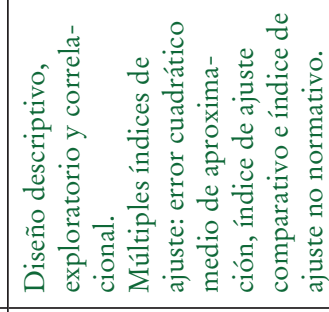 \\
\hline & 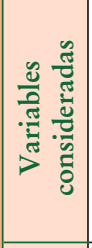 & 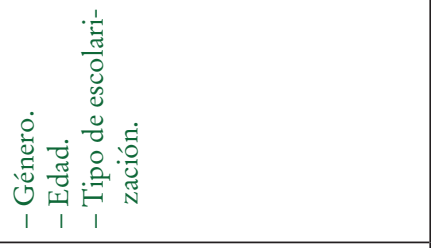 & 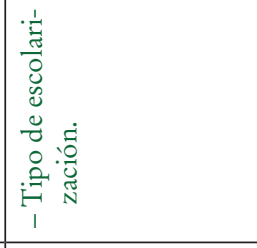 & 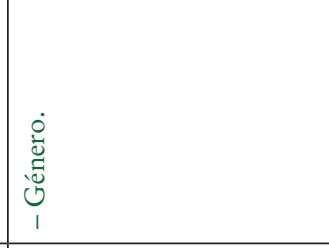 \\
\hline & 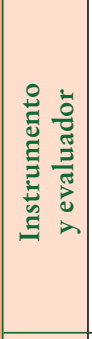 & 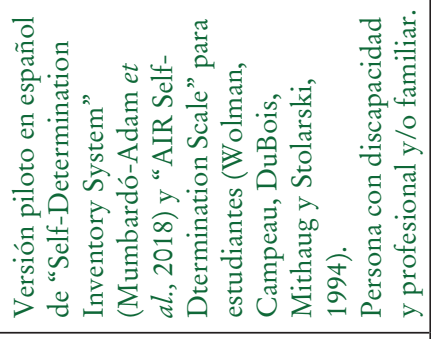 & 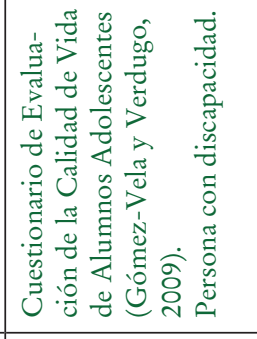 & 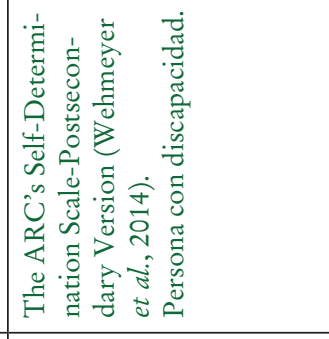 \\
\hline & 㺃 & 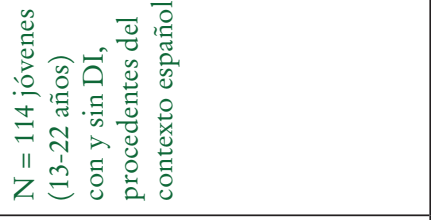 & 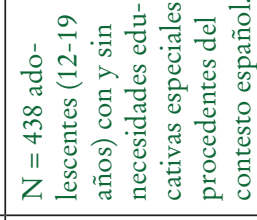 & 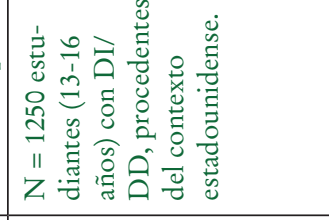 \\
\hline & 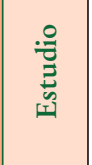 & 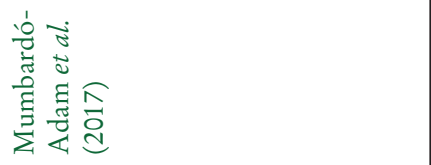 & 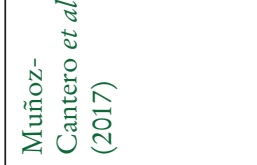 & 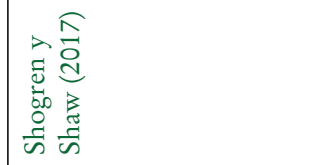 \\
\hline
\end{tabular}

Ediciones Universidad de Salamanca / CC BY-NC-ND

Siglo Cero, vol. 52 (4), 2021, octubre-diciembre, pp. 109-130 
FACTORES PERSONALES Y ESCOLARES SUBYACENTES A LA CONDUCTA AUTODETERMINADA DE NIÑOS Y ADOLESCENTES CON DISCAPACIDAD INTELECTUAL: REVISIÓN DE LA LITERATURA L. BLÁZQUEZ ARRIBAS, M. ${ }^{a}$ I. CALVO ÁlVAREZ Y M. ${ }^{a}$ B. ORGAZ BAZ

\begin{tabular}{|c|c|c|c|c|}
\hline \multirow{6}{*}{ 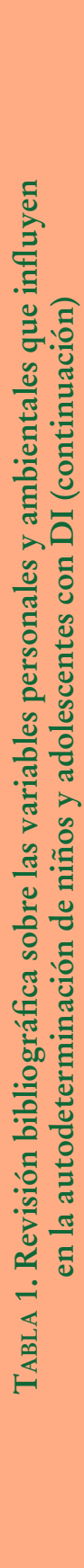 } & 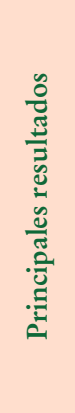 & 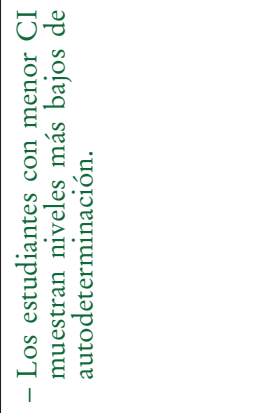 & 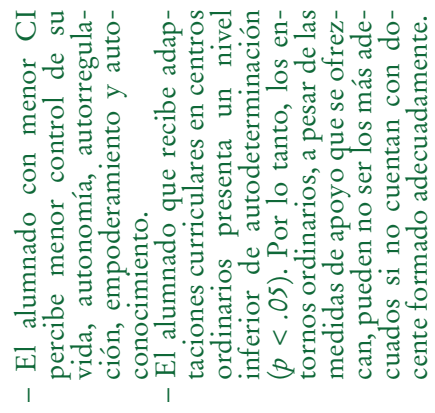 & 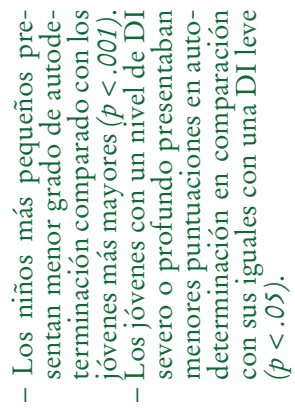 \\
\hline & 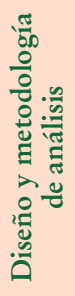 & 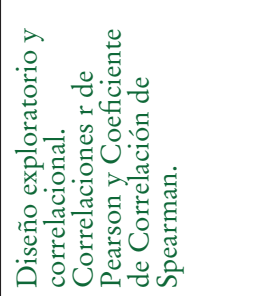 & 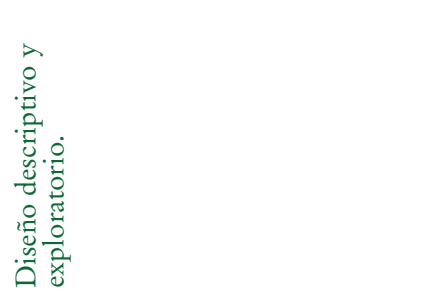 & 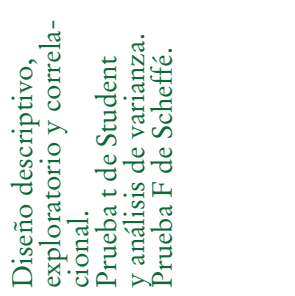 \\
\hline & 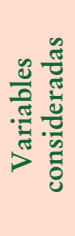 & $\begin{array}{l}\vec{U} \\
1\end{array}$ & 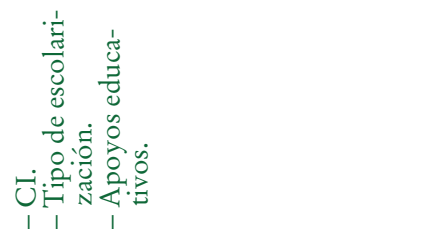 & \\
\hline & 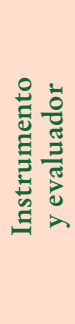 & 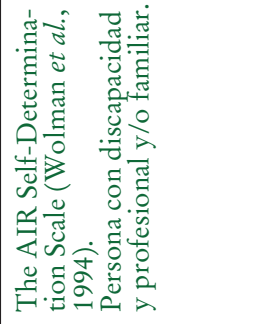 & 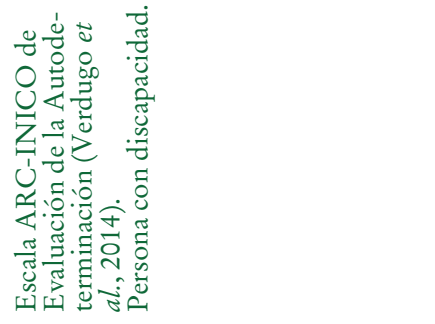 & 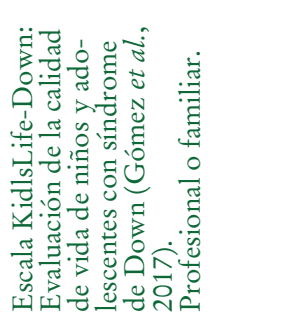 \\
\hline & 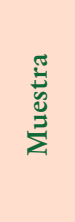 & 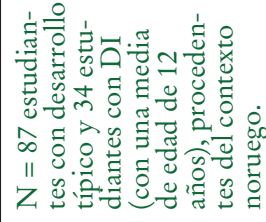 & 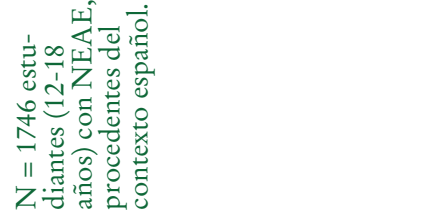 & 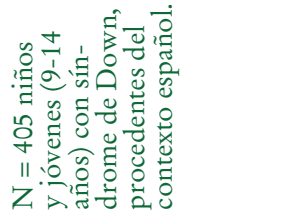 \\
\hline & 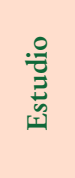 & 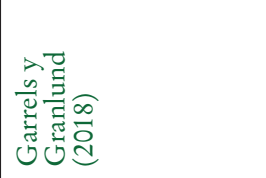 & 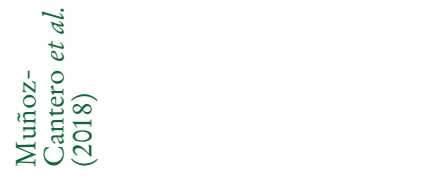 & 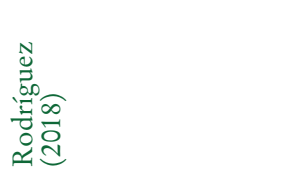 \\
\hline
\end{tabular}

Ediciones Universidad de Salamanca / CC BY-NC-ND

Siglo Cero, vol. 52 (4), 2021, octubre-diciembre, pp. 109-130 
FACTORES PERSONALES Y ESCOLARES SUBYACENTES A LA CONDUCTA AUTODETERMINADA DE NIÑOS Y ADOLESCENTES CON DISCAPACIDAD INTELECTUAL: REVISIÓN DE LA LITERATURA L. BLÁZQUEZ ARRIBAS, M. ${ }^{a}$ I. CALVO ÁlVAREZ Y M. ${ }^{a}$ B. ORGAZ BAZ

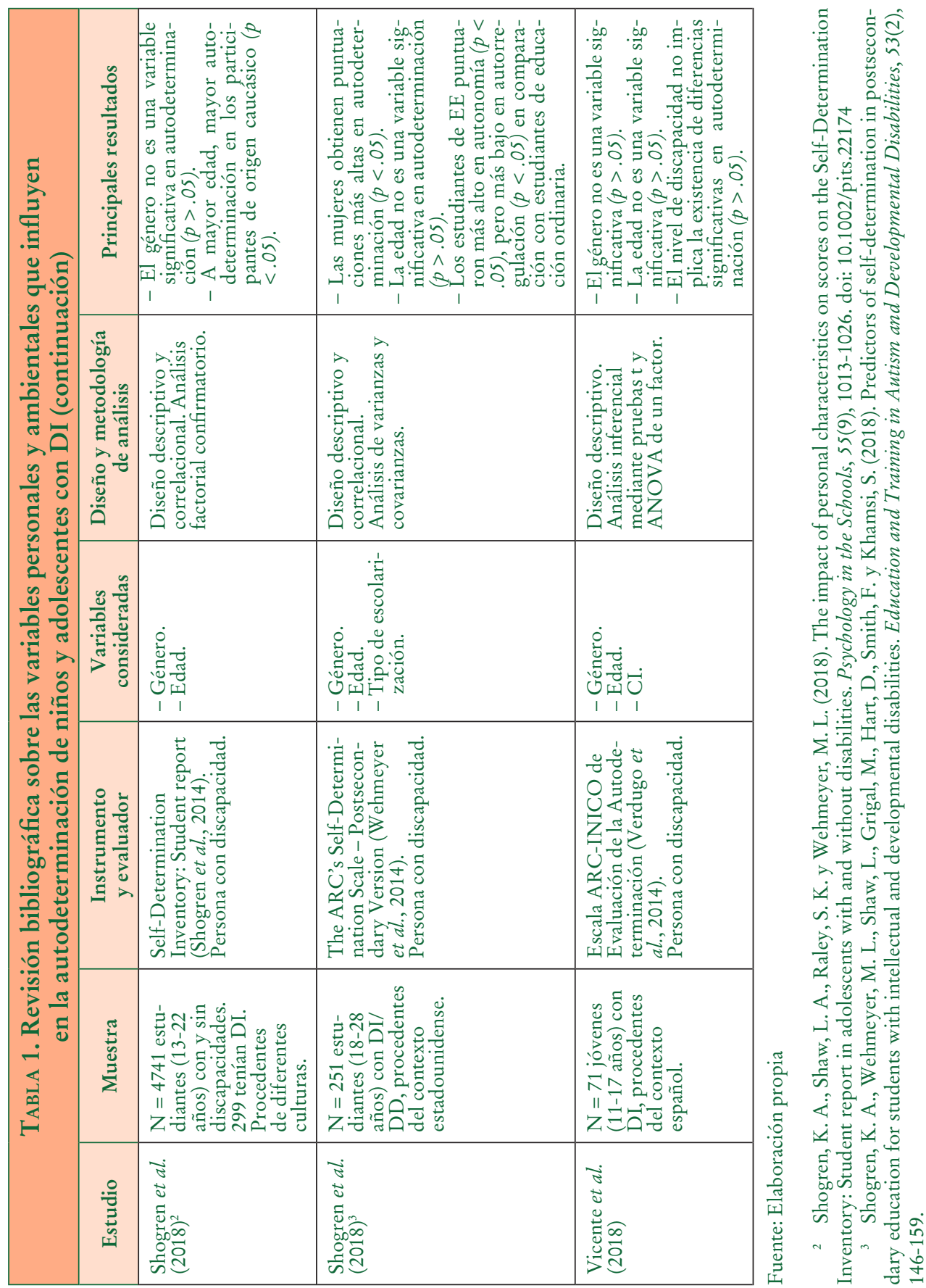

Ediciones Universidad de Salamanca / CC BY-NC-ND

Siglo Cero, vol. 52 (4), 2021, octubre-diciembre, pp. 109-130 
FACTORES PERSONALES Y ESCOLARES SUBYACENTES A LA CONDUCTA AUTODETERMINADA DE NIÑOS Y ADOLESCENTES CON DISCAPACIDAD INTELECTUAL: REVISIÓN DE LA LITERATURA L. BLÁZQUEZ ARRIBAS, M. ${ }^{a}$ I. CALVO ÁlVAREZ Y M. ${ }^{a}$ B. ORGAZ BAZ

\begin{tabular}{|c|c|c|c|c|}
\hline \multirow{6}{*}{ 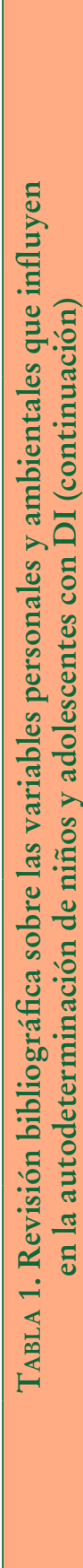 } & 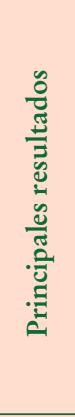 & 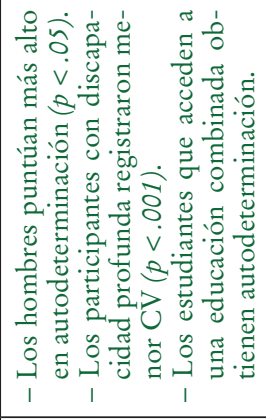 & 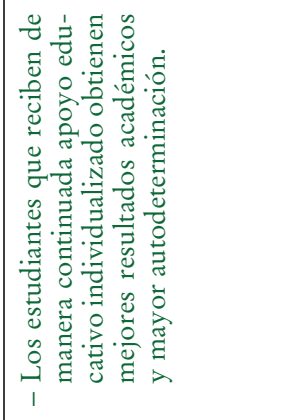 & 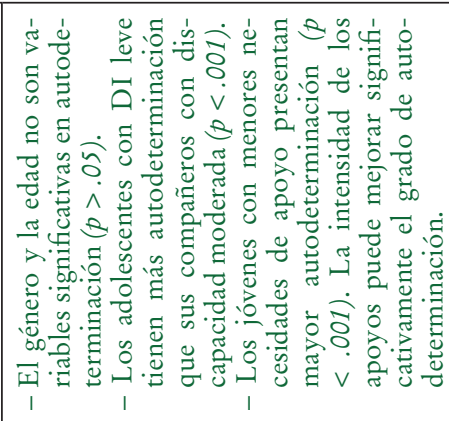 \\
\hline & 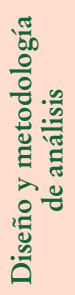 & 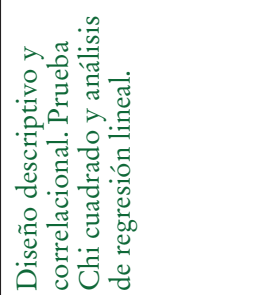 & 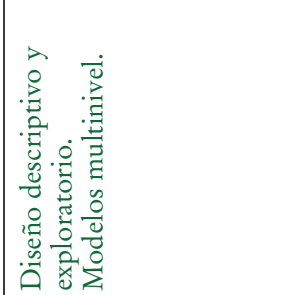 & 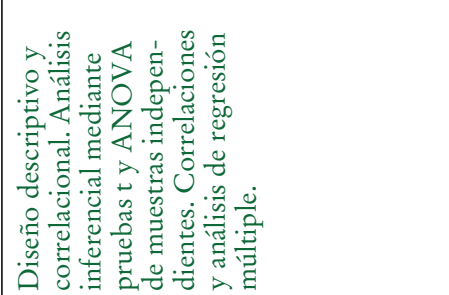 \\
\hline & 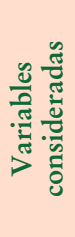 & 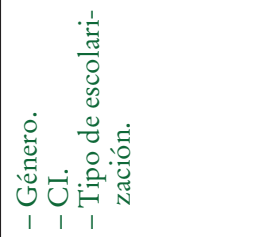 & 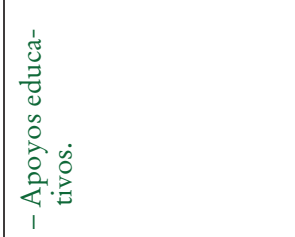 & 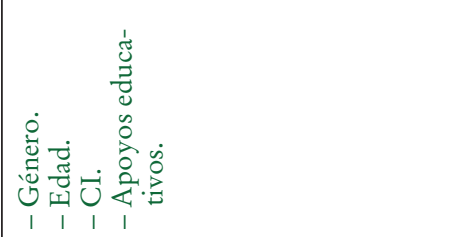 \\
\hline & 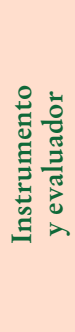 & 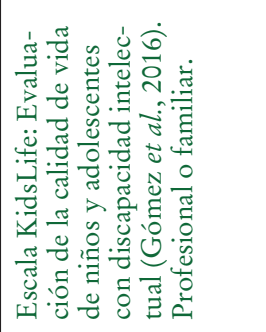 & 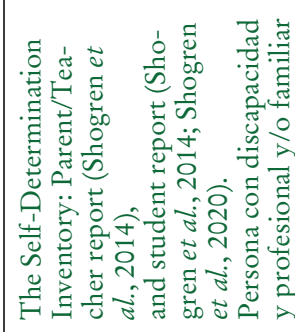 & 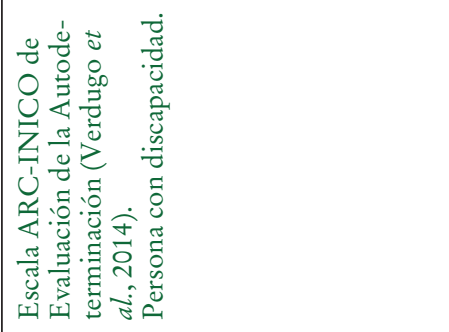 \\
\hline & 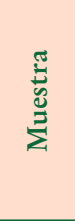 & 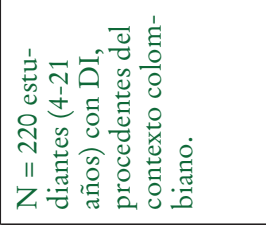 & 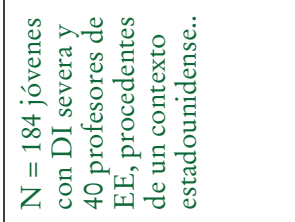 & 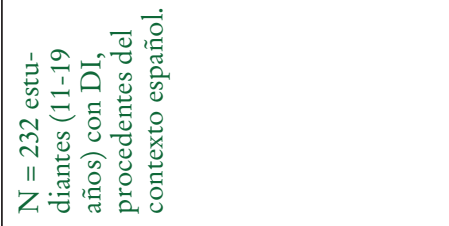 \\
\hline & 异 & 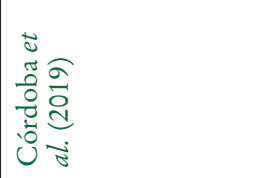 & 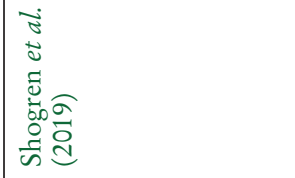 & 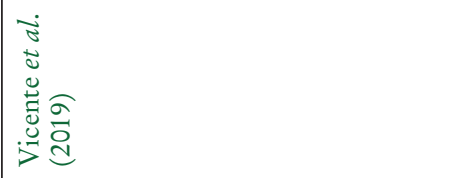 \\
\hline
\end{tabular}

Ediciones Universidad de Salamanca / CC BY-NC-ND

Siglo Cero, vol. 52 (4), 2021, octubre-diciembre, pp. 109-130 
FACTORES PERSONALES Y ESCOLARES SUBYACENTES A LA CONDUCTA AUTODETERMINADA DE NIÑOS Y ADOLESCENTES CON DISCAPACIDAD INTELECTUAL: REVISIÓN DE LA LITERATURA L. BLÁZQUEZ ARRIBAS, M. ${ }^{a}$ I. CALVO ÁlVAREZ Y M. ${ }^{a}$ B. ORGAZ BAZ

\begin{tabular}{|c|c|c|c|c|}
\hline \multirow{6}{*}{ 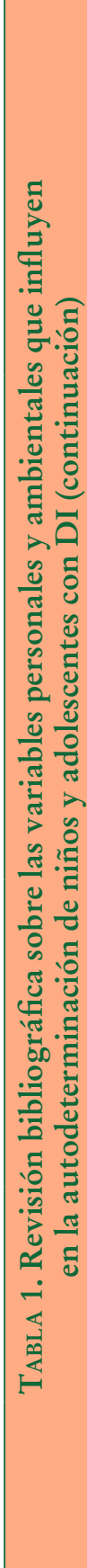 } & 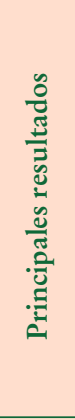 & 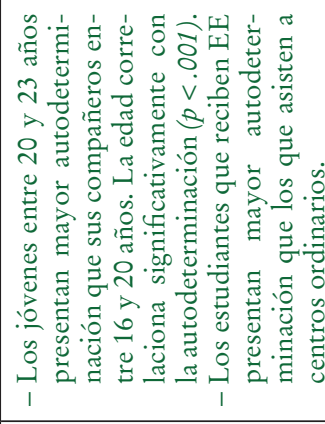 & 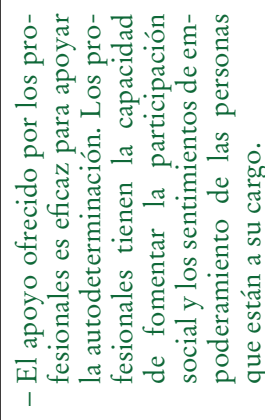 & 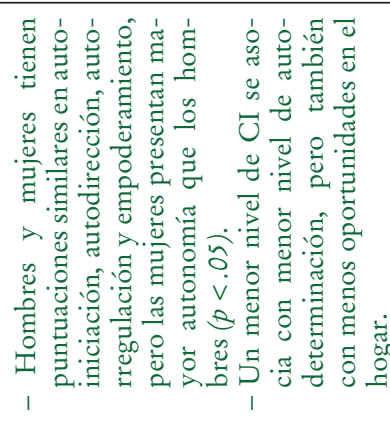 \\
\hline & 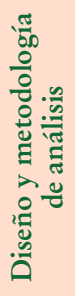 & 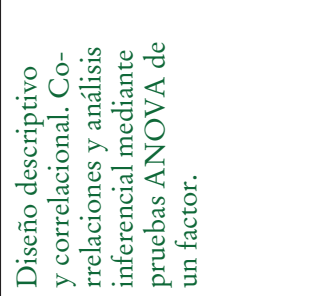 & 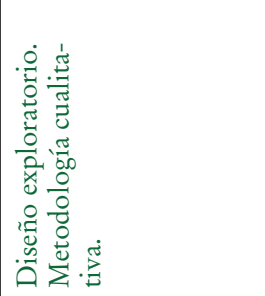 & 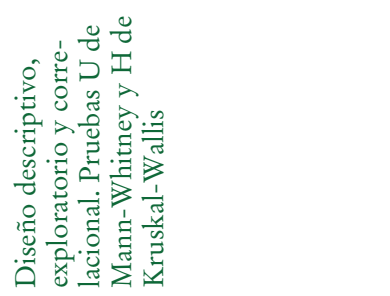 \\
\hline & 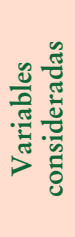 & 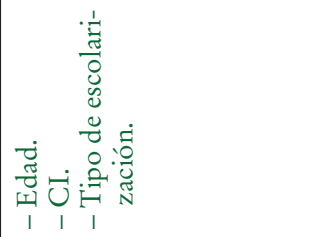 & 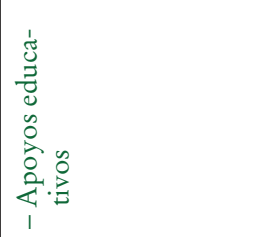 & 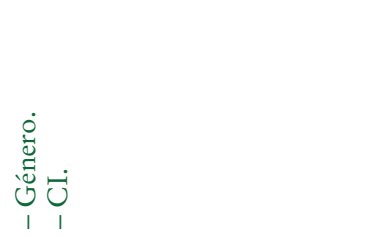 \\
\hline & 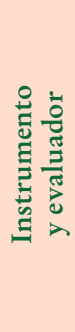 & 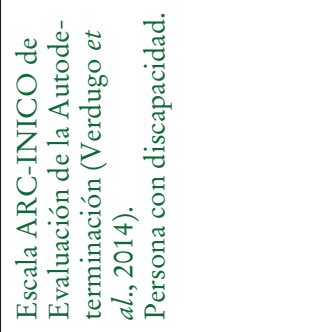 & 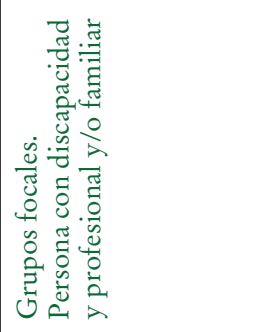 & 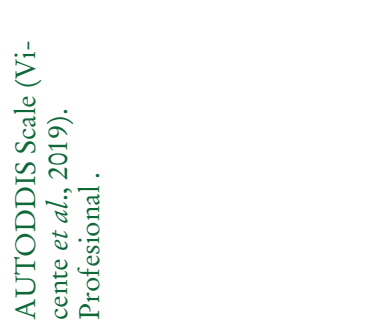 \\
\hline & 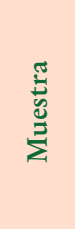 & 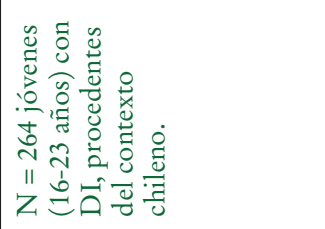 & 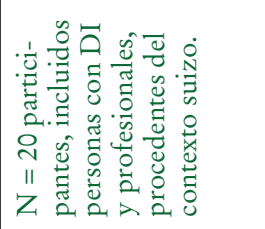 & 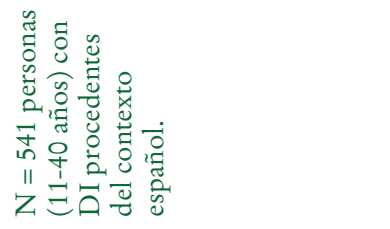 \\
\hline & 莺 & 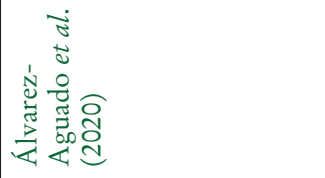 & 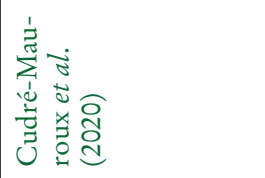 & 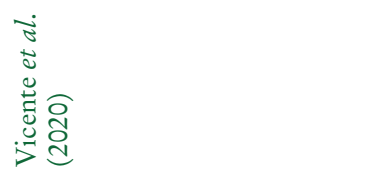 \\
\hline
\end{tabular}


FACTORES PERSONALES Y ESCOLARES SUBYACENTES A LA CONDUCTA AUTODETERMINADA

DE NIÑOS Y ADOLESCENTES CON DISCAPACIDAD INTELECTUAL: REVISIÓN DE LA LITERATURA L. BLÁZQUEZ ARRIBAS, M. ${ }^{\mathrm{a}}$ I. CALVO ÁLVAREZ Y M. ${ }^{\mathrm{a}} \mathrm{B}$. ORGAZ BAZ

\subsection{Variables personales: género, edad y CI}

Los estudios seleccionados analizan la influencia de variables demográficas sobre la conducta autodeterminada, como el género, la edad y el cociente intelectual (en adelante $\mathrm{CI})$.

El $19 \%$ de los estudios revisados concluyen que la variable género no es un factor significativo que interceda en las puntuaciones de autodeterminación (MumbardóAdam et al., 2017; Shogren et al., 2018; Vicente et al., 2018; Vicente et al., 2019). Sin embargo, el $28 \%$ de las investigaciones señalan diferencias dispares entre hombres y mujeres: el $19 \%$ indica que las mujeres obtienen puntuaciones más elevadas en autodeterminación (Cavendish, 2017; Losada-Puente, 2017; Shogren et al., 2017; Vicente et al., 2020) y el $9 \%$ de las investigaciones explican que son los hombres los que tienen mayor número de conductas autodeterminadas (Córdoba et al., 2019; Shogren y Shaw, 2017).

Del mismo modo, el $19 \%$ de los estudios coinciden en que la edad no es una variable importante al no encontrar diferencias significativas entre grupos (Cavendish, 2017; Shogren et al., 2018; Vicente et al., 2018; Vicente et al., 2019). No obstante, el $38 \%$ de las investigaciones señalan una tendencia de que las personas de mayor edad obtienen puntuaciones más elevadas en autodeterminación, probablemente debido a que lo han practicado a lo largo de la infancia (Álvarez-Aguado et al., 2020; Kedrova y Matantseva, 2016; Losada-Puente, 2017; Mumbardó-Adam et al., 2017; Rodríguez, 2018; Shogren et al., 2018). Este hallazgo fue respaldado por autores que explican que los adolescentes más mayores, que trabajan desde una perspectiva de futuro, obtienen puntuaciones superiores en autodeterminación con mayor énfasis en las estrategias de autorrealización (Shogren et al., 2016; Shogren et al., 2015).

Vicente et al. (2018) niegan la existencia de una relación significativa entre el CI de una persona y su grado de autodeterminación. No obstante, la mayoría de investigaciones descubren que las personas con discapacidades leves (o con un funcionamiento intelectual más alto) consiguen ser más autodeterminadas, es decir, hay estudios que defienden que el CI es un aspecto relevante para la autodeterminación (Cavendish, 2017; Córdoba et al., 2019; Garrels y Granlund, 2018; Muñoz-Cantero et al., 2018; Vicente et al., 2019; Vicente et al., 2020). Por ejemplo, Córdoba et al. (2019) y Vicente et al. (2019) concluyen que los estudiantes con niveles moderados o graves de DI obtuvieron puntuaciones significativamente más bajas en autodeterminación que sus compañeros con DI leve. A su vez, Garrels y Granlund (2018) demuestran que los jóvenes con DI, y, por lo tanto, con menor CI en comparación con sus iguales sin dicha discapacidad, muestran menor grado de autodeterminación. Específicamente, Muñoz-Cantero et al. (2018) demuestran que los estudiantes con DI perciben menor control de su vida y menor autonomía, autorregulación, empoderamiento y autoconocimiento respecto al alumnado sin discapacidad.

Sin embargo, la relación existente entre ambas variables es compleja y puede no ser significativa cuando se tienen en cuenta otros factores contextuales (Muñoz-Cantero et al., 2017; Vicente et al., 2019; Vicente et al., 2020); por ejemplo, factores como la edad, los apoyos educativos, las actitudes y creencias sobre discapacidad y autodeter- 
FACTORES PERSONALES Y ESCOLARES SUBYACENTES A LA CONDUCTA AUTODETERMINADA

DE NIÑOS Y ADOLESCENTES CON DISCAPACIDAD INTELECTUAL: REVISIÓN DE LA LITERATURA L. BLÁZQUEZ ARRIBAS, M. ${ }^{a}$ I. CALVO ÁlVAREZ Y M. ${ }^{a}$ B. ORGAZ BAZ

minación o las oportunidades para elegir y tomar decisiones. En definitiva, cualquier persona, a través de la instrucción y de los apoyos necesarios, puede adquirir las habilidades y capacidades que le permita tomar el control, decidir y responsabilizarse de sus acciones (Muñoz-Cantero et al., 2018).

\subsection{Variables ambientales: tipo de escolarización y apoyos educativos}

Existe un amplio conjunto de factores externos a la persona que pueden influir de alguna manera en su grado de autodeterminación, algunos de ellos facilitan y otros limitan las posibilidades de aprendizaje de habilidades relacionadas con la autodeterminación. Los estudios seleccionados hacen referencia al tipo de escolarización y a los apoyos educativos ofrecidos.

Algunos autores creen importante valorar el tipo de escolarización: educación ordinaria o educación especial (en adelante, EE). Shogren et al. (2018) explican que aquellos estudiantes que reciben evaluaciones alternativas propias de la EE puntúan más alto en autonomía, pero obtienen puntuaciones más bajas en autorregulación, en comparación con los estudiantes que reciben evaluaciones regulares propias de la educación ordinaria.

El $14 \%$ de los estudios revisados defienden que las escuelas segregadas ofrecen escasas oportunidades para elegir y practicar habilidades propias de la autodeterminación y, por ello, los estudiantes que están matriculados más tiempo en entornos de educación ordinaria muestran mayor número de veces conductas autodeterminadas en comparación con aquellos alumnos que están más tiempo en entornos segregados (Losada-Puente, 2017; Muñoz-Cantero et al., 2017; Shogren et al., 2016).

Sin embargo, otro $14 \%$ precisa que la escolarización ordinaria puede no ser la más adecuada para promover la autodeterminación debido a la formación de sus docentes, que, en ocasiones, no es la apropiada (Álvarez-Aguado et al., 2020; Mumbardó-Adam et al., 2017; Muñoz-Cantero et al., 2018). En este sentido, la formación docente cobra mayor relevancia que el tipo de centro educativo. Muñoz-Cantero et al. (2017) concluyen que la escolarización ordinaria influye positivamente en la autodeterminación, pero, un año más tarde, Muñoz-Cantero et al. (2018) especifican que dicha escolarización es la adecuada siempre y cuando cuente con un equipo docente formado tanto en discapacidad como en metodologías para promover la autodeterminación.

Según Mumbardó-Adam et al. (2017), los centros de EE están más formados para promover la autodeterminación y los centros ordinarios necesitan considerar otras formas de trabajo para favorecer la inclusión educativa y garantizar la CV del alumnado.

A pesar de estas diferencias, cabe mencionar una tipología alternativa denominada educación combinada. Según Córdoba et al. (2019), los estudiantes que acceden a esta modalidad obtienen mayor puntuación en autodeterminación en comparación con los compañeros que asisten a una escuela ordinaria, probablemente debido a que se benefician de los aspectos positivos de ambas escolarizaciones. Para ellos esta tipolo- 
FACTORES PERSONALES Y ESCOLARES SUBYACENTES A LA CONDUCTA AUTODETERMINADA

DE NIÑOS Y ADOLESCENTES CON DISCAPACIDAD INTELECTUAL: REVISIÓN DE LA LITERATURA L. BLÁZQUEZ ARRIBAS, M. ${ }^{a}$ I. CALVO ÁlVAREZ Y M. ${ }^{a}$ B. ORGAZ BAZ

gía puede ser la alternativa perfecta para permitir que los estudiantes con DI adquieran las competencias necesarias para su desarrollo personal y su autodeterminación.

Independientemente del tipo de escolarización que se ofrezca, los estudios hacen hincapié en los apoyos educativos para promover la participación y fomentar la conducta autodeterminada. Las personas con DI están en mejores condiciones de lograr la autodeterminación cuando reciben el apoyo que necesitan en el momento adecuado (Cavendish, 2017; Cudré-Mauroux et al., 2020; Shogren et al., 2016; Shogren et al., 2019; Shogren y Shaw, 2016). Los apoyos inciden en el éxito académico y en la implicación en la toma de decisiones educativas, además mejoran el bienestar, la autorregulación, la autonomía e independencia, la inclusión socioeducativa, la transición a la vida adulta, la consecución de empleo normalizado y, en general, una mayor CV (Shogren y Shaw, 2016).

Para Muñoz-Cantero et al. (2018), los apoyos educativos deben configurarse como una red de relaciones, coordinación e interdependencia entre docentes y familiares, quienes deben aunar sus esfuerzos para favorecer la promoción de la autodeterminación desde la adolescencia. Vicente et al. (2019) sugieren que los apoyos ofrecidos por la escuela son una variable con mayor significación que, por ejemplo, el grado de discapacidad del alumno o el tipo de escolarización que esté recibiendo. La importancia no recae meramente en las características personales del estudiante o en la tipología del centro escolar, sino que la intensidad, el tipo y la frecuencia de los apoyos se convierten en variables significativas que influyen en el grado de autodeterminación de cada niño o adolescente con discapacidad.

\section{Discusión y conclusión de los resultados}

Los resultados que se pueden inferir de esta revisión expresan que la autodeterminación está influenciada tanto por variables personales como por variables ambientales, no obstante, existe discrepancia en los resultados.

En relación al género, los resultados son heterogéneos. Hay que tener en cuenta que se tratan diferentes contextos culturales y, probablemente, sea lo que explique la gran variedad de resultados (Rodríguez y Cavendish, 2013; Shogren et al., 2013). Aun así, existen diferencias en el contexto español, de tal modo que algunos autores niegan la influencia del género en la autodeterminación (Mumbardó-Adam et al., 2017; Vicente et al., 2018; Vicente et al., 2019), mientras que hay estudios que indican mayores puntuaciones en las mujeres respecto a los hombres (Gómez-Vela et al., 2012; Losada-Puente, 2017).

La mayoría de estudios que tienen en cuenta la edad como variable concluyen que, a mayor edad, mayor autodeterminación, siempre y cuando se practique durante la infancia y adolescencia temprana para facilitar la transición a la vida adulta (Carter et al., 2013; Shogren et al., 2013).

Respecto al CI, las investigaciones describen resultados dispares, hay investigaciones que confirman (Carter et al., 2013) y otras que niegan (Gómez-Vela et al., 2012; Verdugo et al., 2012) la relación entre el funcionamiento intelectual y la autodetermi- 
FACTORES PERSONALES Y ESCOLARES SUBYACENTES A LA CONDUCTA AUTODETERMINADA

DE NIÑOS Y ADOLESCENTES CON DISCAPACIDAD INTELECTUAL: REVISIÓN DE LA LITERATURA L. BLÁZQUEZ ARRIBAS, M. ${ }^{a}$ I. CALVO ÁlVAREZ Y M. ${ }^{a}$ B. ORGAZ BAZ

nación. El trabajo de Verdugo et al. (2012) especifica que el CI no señala realmente la capacidad para llegar a ser autodeterminado, sino que el CI puede determinar dónde vive, estudia o trabaja la persona, y dichos contextos son los que ofrecen o limitan las oportunidades para actuar de manera autodeterminada. Maes et al. (2007) explicaron que la escuela y la comunidad ofrecen mayores oportunidades para practicar la autodeterminación y mejorar el desarrollo personal a aquellas personas con mayores niveles de comunicación y de habilidades intelectuales. Esta situación es explicada por Navas et al. (2017), comparándolo con "un círculo vicioso en el que no se otorgan oportunidades para la elección y participación a aquellas personas con más dificultades, lo que genera a su vez que estas últimas se perpetúen y sigan limitando oportunidades futuras de participación y elección" (p. 36). Vicente et al. (2020) entienden que la capacidad de actuar de manera autodeterminada depende tanto de las capacidades personales como de las oportunidades que el entorno puede ofrecer para que estos comportamientos se implementen con eficacia.

Por todo lo anterior, todas las variables que pueden influir en la autodeterminación deben ser contrastadas con otras variables contextuales, ya que la autodeterminación se desarrolla en una interacción constante entre la persona y el entorno en el que se desenvuelve (Baña-Castro et al., 2015). Por lo tanto, variables como el género (Rodríguez y Cavendish, 2013) o el CI (Verdugo et al., 2012) pueden no ser significativas si se tienen en cuenta otros factores contextuales, por ejemplo, la cultura, la formación y las actitudes docentes o la intensidad y la frecuencia de los apoyos educativos que el alumno recibe.

Las investigaciones centradas en el ambiente escolar también muestran resultados ambiguos respecto al tipo de escolarización, algunas entienden la escolarización ordinaria como el mejor entorno para promover la autodeterminación (Carter et al., 2013) y otras, en cambio, se decantan por la EE (Shogren et al., 2007). Esta variable puede estar relacionada con el grado de discapacidad de los estudiantes, entendiendo que aquellos con discapacidades leves tienen más oportunidades de acceder a entornos ordinarios; no obstante, Shogren et al. (2015) defendieron que incluso el alumnado con discapacidades graves obtiene puntuaciones más elevadas en autodeterminación cuando asiste a una escuela ordinaria.

Sin embargo, más allá de la modalidad de escolarización, lo realmente importante es ofrecer una educación adecuada y ajustada a las necesidades individuales que prepare a los estudiantes para la autodeterminación y para la transición a la vida adulta e independiente (Kislyakov, 2017). Por ello, las estrategias y la formación docente, como fomentar la autonomía en las tareas escolares (Shogren et al., 2015) o crear oportunidades de autodefensa (Wehmeyer y Abery, 2013), reducir la ratio del aula (Muñoz-Cantero y Losada-Puente, 2018) y ofrecer los apoyos educativos necesarios (Wehmeyer et al., 2012), son variables fundamentales e imprescindibles para promover la autodeterminación en los adolescentes con discapacidad.

Por último, las investigaciones inciden en la importancia de los apoyos, los cuales adquieren una importancia relevante para paliar las discrepancias que puede haber entre lo que la persona es capaz de hacer y las demandas del entorno en el que se desenvuelve (Verdugo y Schalock, 2010). Brock et al. (2020) determinan el apoyo natural 
entre compañeros como un soporte académico y social que permite adquirir los conocimientos y habilidades necesarias para la transición a la vida adulta. Cudré-Mauroux et al. (2020) subrayan la importancia de ofrecer apoyos para tomar decisiones importantes, siempre mostrando respeto por los intereses y preferencias del estudiante. Todas las personas, a través de la instrucción y de los apoyos escolares adecuados, pueden adquirir las habilidades y las capacidades que le permitan tomar el control, decidir y responsabilizarse de sus acciones (DiMaggio et al., 2020; Muñoz-Cantero et al., 2018). Peralta y Arellano (2014) explicaron que "aunque el grado de discapacidad puede influir en la conducta autodeterminada, no es el único factor determinante y siempre es posible alcanzar un cierto grado de autodeterminación, puesto que no es una variable continua" (p. 63). La autodeterminación no supone un proceso o un resultado ni exige tener control total sobre la vida y hacer las tareas por sí solo, sino que puede ser percibida de manera diferente dependiendo de las habilidades e intereses de la persona y puede ser experimentada en diferentes niveles por el mismo individuo a lo largo de su vida.

En definitiva, los resultados de esta revisión permiten conocer qué variables influyen en la autodeterminación para, posteriormente, diseñar, implementar, evaluar y mejorar eficazmente las estrategias, intervenciones y apoyos enfocados en la promoción de la conducta autodeterminada en personas con discapacidad (Wehmeyer et al., 2019).

\subsection{Limitaciones}

A la hora de interpretar los resultados, es importante considerar las limitaciones del estudio: 1) las investigaciones analizadas han sido desarrolladas en diferentes contextos, por lo que no pueden ser generalizados a una muestra determinada; 2) los participantes de los estudios tienen diferentes tipos de discapacidades, por lo que los resultados no pueden centrarse exclusivamente en el colectivo de DI; 3) han utilizado diferentes tamaños de muestra y diferentes instrumentos de evaluación; 4) la mayoría de los trabajos recogen la percepción de la persona con discapacidad sobre su autodeterminación, pero otros estudios recogen la percepción de los profesionales y de la familia; 5) utilizan diferentes método de análisis de datos; 6) únicamente dos investigaciones ha utilizado una metodología cualitativa; 7) la revisión se ha centrado exclusivamente en el contexto educativo. Como futuras líneas de investigación se sugiere seguir estudiando las variables que pueden influir en la conducta autodeterminada de personas con discapacidad para considerar el diseño e implementación de programas de intervención y promoción de la autodeterminación.

\section{Referencias bibliográficas}

Álvarez-Aguado, I., Vega-Córdova, V., Campaña-Vilo, K., González-Carrasco, SpenCer-González, H. y Arriagada-Chinchón, R. (2020). Habilidades de autodetermina- 
FACTORES PERSONALES Y ESCOLARES SUBYACENTES A LA CONDUCTA AUTODETERMINADA DE NIÑOS Y ADOLESCENTES CON DISCAPACIDAD INTELECTUAL: REVISIÓN DE LA LITERATURA L. BLÁZQUEZ ARRIBAS, M. ${ }^{a}$ I. CALVO ÁlVAREZ Y M. ${ }^{a}$ B. ORGAZ BAZ

ción en estudiantes chilenos con discapacidad intelectual: avanzando hacia una inclusión exitosa. Revista Colombiana de Educación, 1(79), 369-394. https://doi.org/10.17227/rce. num79-9663

Baña-Castro, M., Losada-Puente, L. y Muñoz-Cantero, J. M. (2015). Condicionantes personales y ambientales de la conducta autodeterminada en la adolescencia con alteraciones del desarrollo intelectual. Ciencias Psicológicas, 9(3), 283-292.

Brock, M. E., Schaefer, J. M. y Seaman, R. L. (2020). Self-determination and agency for all: Supporting students with severe disabilities. Theory into Practice, 59(2), 162-171. https:// doi.org/10.1080/00405841.2019.1702450

Carter, E. W., Lane, K. L., Cooney, M., Weir, K., Moss, C. K. y MachaliceK, W. (2013). Parent assessments of self-determination importance and performance for students with autism or intellectual disability. American Journal on Intellectual and Developmental Disabilities, 118(1), 16-31. https://doi.org/10.1352/1944-7558-118.1.16

CAVENDish, W. (2017). The role of gender, race/ethnicity and disability status on the relationship between student perceptions of school and family support and self-determination. Career Development and Transition for Exceptional Individuals, 40(2), 113-122. https:// doi.org/10.1177/2165143416629359

Córdoba, L., Salamanca, L. M. y Mora, A. (2019). Calidad de vida en personas con discapacidad intelectual y múltiple de 4 a 21 años de edad. Psychologia, 13(2), 79-93. http://dx.doi. org.ezproxy.usal.es/10.21500/19002386.4014

Cudré-Mauroux, A., Piérart, G. y Vaucher, C. (2020). Partnership with social care professionals as a context for promoting self-determination among people with intellectual disabilities. Research in Developmental Disabilities, 99, 103602. https://doi.org/10.1016/j. ridd.2020.103602

Garrels, V. y Granlund, M. (2018). Measuring self-determination in Norwegian students: adaptation and validation of the AIR self-determination scale. European Journal of Special Needs Education, 33(4), 466-480. https://doi.org/10.1080/08856257.2017.1342420

Gómez-Vela, M., Verdugo, M. Á., GonZÁlez-Gil, F., Badia, M. y Wehmeyer, M. L. (2012). Assessment of self-determination of Spanish students with intellectual and other educational needs. Educational and Training in Autism and Developmental Disabilities, 47(1), 48-57.

Kedrova, I. A. y Matantseva, T. N. (2016). Adolescents with intellectual disabilities: personal aspects of their developmental disability. International Journal of Environmental and Science Education, 11(8), 2003-2014. https://doi.org/10.12973/ijese.2016.573a

KISLYAKOv, P. A. (2017). Risk factors related to antisocial behavior in teenagers with intellectual disabilities. Psychology in Russia: state of the Art, 10(2), 215-227. https://doi.org/10.11621/ pir.2017.0215

Losada-Puente, L. (2017). Autodeterminación y calidad de vida en adolescentes con discapacidad intelectual y otras alteraciones del desarrollo. (Doctorado). Universidad de A Coruña.

Maes, B., Lambrechts, G., Hostyn, I. y Petry, K. (2007). Quality-enhancing interventions for people with profound intellectual and multiple disabilities: a review of the empirical research literature. Journal of Intellectual and Developmental Disability, 32(3), 163-178. https://doi.org/10.1080/13668250701549427

Mumbardó-Adam, C., Guàrdia-Olmos, J. y Giné, C. (2018). Autodeterminación: midiendo el constructo en jóvenes con y sin discapacidad. Siglo Cero, 1, 137-138. https://doi. org/10.14201/scero20180 
FACTORES PERSONALES Y ESCOLARES SUBYACENTES A LA CONDUCTA AUTODETERMINADA DE NIÑOS Y ADOLESCENTES CON DISCAPACIDAD INTELECTUAL: REVISIÓN DE LA LITERATURA L. BLÁZQUEZ ARRIBAS, M. ${ }^{a}$ I. CALVO ÁlVAREZ Y M. ${ }^{a}$ B. ORGAZ BAZ

Mumbardó-Adam, C., Shogren, K., Guàrdia-Olmos, J. y Giné, C. (2017). Contextual predictors of self-determined actions in students with and without intellectual disability. Psychology in the Schools, 54(2), 183-195. https://doi.org/10.1002/pits.21987

Muñoz-Cantero, J. M. y Losada-Puente, L. (2018). Implicaciones de la actitud docente en la calidad de vida del alumnado con alteraciones del desarrollo intelectual. Educación XXI, 21(2), 37-58. https://doi.org/10.5944/educxx1.19535

Muñoz-Cantero, J. M., Losada-Puente, L. y Almeida, L. S. (2017). Quality of life, adolescence and inclusive schools: comparing regular and special needs students. Bordón, 69(1), $139-154$.

Muñoz-Cantero, J. M., Losada-Puente, L. y Espiñeira-Bellón, E. M. (2018). Apoyos y autodeterminación en la escuela inclusiva. Factores relacionados con la obtención de resultados personales positivos. Revista Española de Orientación y Psicopedagogía, 29(1), 8-24. https://doi.org/10.5944/reop.vol.29.num.1.2018.23291

Navas, P., Verdugo, M. Á., Martínez, S., Sainz, F. y Aza, A. (2017). Derechos y calidad de vida en personas con discapacidad intelectual y mayores necesidades de apoyo. Siglo Cero, 48(4), 7-66. https://doi.org/10.14201/scero2017484766

Peralta, F. y Arellano, A. (2014). La autodeterminación de las personas con discapacidad intelectual: situación actual en España. Revista CES Psicología, 7(2), 59-77.

Rodríguez, M. M. (2018). Evaluación de la calidad de vida en niños y jóvenes con síndrome de Down. (Doctorado). Universidad de Salamanca.

Rodríguez, R. J. y Cavendish, W. (2013). Differences in the relationship between family environments and self-determination among Anglo, Latino, and female students with disabilities. Career Development and Transition for Exceptional Individuals, 36(3), 152-162. https://doi.org/10.1177/2165143412461524

SCHALOCK, R. L. (2010). Aplicaciones del paradigma de calidad de vida a las personas con discapacidad intelectual y del desarrollo. En M. Á. Verdugo, M. Crespo y T. Nieto, Aplicación del paradigma de calidad de vida. VII Seminario de Actualización Metodológica en Investigación sobre Discapacidad SAID (pp. 11-18). INICO.

SCHALOCK, R. L. y VERDUGO, M. Á. (2002/2003). Quality of life for human service practitioners. American Association on Mental Retardation.

Shogren, K. A., Burke, K. M., Antosh, A. A., Wehmeyer, M. L., Laplante, T., Shaw, L. A. y RALEY, S. K. (2019). Impact of the Self-Determined Learning Model of Instruction on SelfDetermination and Goal Attainment in adolescents with intellectual disability. Journal of Disability Policy Studies, 30(1), 22-34. https://doi.org/10.1177/1044207318792178

Shogren, K. A., Garnier-Villarreal, M., Dowsett, C. y Little, T. D. (2016). Exploring student, family and school predictors of self-determination using NLTS2 Data. Career Development and Transition for Exceptional Individuals, 39(1), 23-33. https://doi. org $/ 10.1177 / 2165143414546685$

Shogren, K. A., Mccart, A. B., Lyon, K. J. y Sailor, W. S. (2015). All means all: building knowledge for inclusive school wide transformation. Research and Practice for Persons with Severe Disabilities, 40(3), 173-191. https://doi.org/10.1177/1540796915586191

Shogren, K. A. y SHAw, L. A. (2016). The role of autonomy, self-realization and psychological empowerment in predicting early adulthood outcomes for youth with disabilities. Remedial and Special Education, 37(1), 55-62. https://doi.org/10.1177/0741932515585003

Shogren, K. A. y SHAw, L. A. (2017). The impact of personal factors on self-determination and early adulthood outcome constructs in youth with disabilities. Journal of Disability Policy Studies, 27(4), 223-233. https://doi.org/10.1177/1044207316667732

Ediciones Universidad de Salamanca / CC BY-NC-ND

Siglo Cero, vol. 52 (4), 2021, octubre-diciembre, pp. 109-130 
FACTORES PERSONALES Y ESCOLARES SUBYACENTES A LA CONDUCTA AUTODETERMINADA

DE NIÑOS Y ADOLESCENTES CON DISCAPACIDAD INTELECTUAL: REVISIÓN DE LA LITERATURA L. BLÁZQUEZ ARRIBAS, M. ${ }^{a}$ I. CALVO ÁlVAREZ Y M. ${ }^{a}$ B. ORGAZ BAZ

Shogren, K. A., Shaw, L. A., Raley, S. K. y Wehmeyer, M. L. (2018). The impact of personal characteristics on scores on the Self-Determination Inventory: student report in adolescents with and without disabilities. Psychology in the Schools, 55(9), 1013-1026. https://doi. org/10.1002/pits. 22174

Shogren, K. A., Wehmeyer, M. L. y Burke, M. (2017). Self-determination. En K. A. Shogren, M. L. WehmeYer y N. N. Singh (Eds.), Handbook of positive psychology in intellectual and developmental disabilities. Translating research into practice (pp. 49-64). Springer Series on Child and Family Studies.

Shogren, K. A., Wehmeyer, M. L., Palmer, S. B. y Paek, Y. (2013). Exploring personal and school environment characteristics that predict self-determination. Exceptionality, 21(3), 147-157. https://doi.org/10.1080/09362835.2013.802231

Shogren, K. A., Wehmeyer, M. L., Palmer, S. B., Forber-Pratt, A., Little, T. y López, S. (2015). Causal Agency Theory: reconceptualizing a functional model of self-determination. Education and Training in Autism and Developmental Disabilities, 50(3), 251-263.

Shogren, K. A., Wehmeyer, M. L., Palmer, S. B., Rifenbark, G. G. y LitTle, T. D. (2015). Relationships between self-determination and postschool outcomes for youth with disabilities. The Journalof SpecialEducation, 48(4),256-267. https://doi.org/10.1177/0022466913489733

Shogren, K. A., Wehmeyer, M. L., Palmer, S. B., Soukup, J. H., Little, T., Garner, N. y LAWRENCE, M. (2007). Examining individual and ecological predictors of self-determination of student with disability. Exceptional Children, 73(4), 488-509. https://doi. org/10.1177/001440290707300406

Shogren, K. A., Wehmeyer, M. L., Shaw, L., Grigal, M., Hart, D., Smith, F. y Khamsi, S. (2018). Predictors of self-determination in postsecondary education for students with intellectual and developmental disabilities. Education and Training in Autism and Developmental Disabilities, 53(2), 146-159.

UnESCO. (2016). Educación 2030: Declaración de Incheon y Marco de Acción para la realización del Objetivo de Desarrollo Sostenible 4: Garantizar una educación inclusiva y equitativa de calidad y promover oportunidades de aprendizaje permanente para todos. Recuperado de: https://unesdoc.unesco.org/ark:/48223/pf0000245656_spa

Verdugo, M. Á., Gómez-Vela, M., Vicente, E., Pulido, R. F., Badia, M., González-Gil, F. y Calvo, M. I. (2012). Evaluación de la conducta autodeterminada de alumnos con discapacidad intelectual y dificultades de aprendizaje. En M. Á. Verdugo, R. Canal, C. Jerano, M. Badia y A. L. Aguado (Eds.), Aplicación del paradigma de calidad de vida a la intervención con personas con discapacidad desde una perspectiva integral (pp. 55-66). INICO.

Verdugo, M. Á. y Schalock, R. L. (2010). Últimos avances en el enfoque y concepción de las personas con discapacidad intelectual. Siglo Cero, 41(4), 7-21.

Vicente, E., Guillén-Martín, V. M., Verdugo, M. Á. y Calvo, M. I. (2018). El rol de los factores personales y familiares en la autodeterminación de jóvenes con discapacidad intelectual. Psicología Educativa, 24(2), 75-83. https://doi.org/10.5093/psed2018a13

Vicente, E., Mumbardó-Adam, C., Guillén, V. M., Coma-Roselló, T., Bravo-Álvarez, M. A. y SÁNCHEZ, S. (2020). Self-Determination in people with intellectual disability: the mediating role of opportunities. International Journal of Environmental Research and Public Health, 17, 6201. https://doi.org/10.3390/ijerph17176201

Vicente, E., Verdugo, M. Á., Gómez-Vela, M., Fernández-Pulido, R., Wehmeyer, M. L. y Guillén, M. V. (2019). Personal characteristics and school contextual variables associated with student self-determination in Spanish context. Journal of Intellectual and Developmental Disability, 44(1), 23-34. https://doi.org/10.3109/13668250.2017.1310828

Ediciones Universidad de Salamanca / CC BY-NC-ND

Siglo Cero, vol. 52 (4), 2021, octubre-diciembre, pp. 109-130 
Vicente, E., Verdugo, M. Á., Guillén, V. M., Martínez-Molina, A., Gómez, L. E. e Ibáñez, A. (2020). Advances in the assessment of self-determination: internal structure of a scale for people with intellectual disabilities aged 11 to 40. Journal of Intellectual Disability Research, 64(9), 700-712. https://doi /10.1111/jir.12762

Wehmeyer, M. L. (2006). Factores intraindividuales y medioambientales que afectan a la autodeterminación. Siglo Cero, 37(4), 47-56.

Wehmeyer, M. L. (2020). Self-determination in adolescents and adults with intellectual and developmental disabilities. Current Opinion in Psychiatry, 33(2), 81-85. https://doi. org/10.1097/YCO.0000000000000576

Wehmeyer, M. L. y Abery, B. H. (2013). Self-determination and choice. Intellectual and Developmental Disabilities, 51(5), 399-411.

Wehmeyer, M. L., Field, S. y Thoma, C. A. (2012). Self-determination and adolescent transition education. En M. L. WeHmeyer y K. W. WebB (Eds.), Handbook of adolescent transition education for youth with disabilities (pp. 171-190). Routledge.

Wehmeyer, M. L., Nota, L., Soresi, S., Shogren, K. A., Momingstar, M. E., Ferrari, L. ... Dimaggio, I. (2019). A crisis in career development: life designing and implications for transition. Career Development and Transition for Exceptional Individuals, 42(3), 179-187. https://doi.org/10.1177/2165143417750092 